\title{
Influence of electromigration field on the step bunching process on $\mathrm{Si}(111)$
}

\author{
V. Usov, ${ }^{*}$ C. O Coileain, and I. V. Shvets \\ Centre for Research on Adaptive Nanostructures and Nanodevices (CRANN), School of Physics, Trinity College, Dublin 2, Ireland
}

(Received 10 February 2010; revised manuscript received 26 June 2010; published 6 October 2010)

\begin{abstract}
We managed to isolate the effects of electromigration in the dynamics of the step bunching process on the vicinal $\mathrm{Si}(111)$ surface. Unlike in conventional experiments, we conducted the annealing of $\operatorname{Si}(111)$ in a specially engineered setup enabling independent temperature control and an in-plane electric field. The primary result is that the step bunching process continues to take place at relatively low applied electric fields and ceases below $E=0.5 \mathrm{~V} / \mathrm{cm}$. Reduction in the electric field results in a significant expansion of step bunches width and elongation of the crossing steps running along the terraces. A theoretically predicted systematic increase in the number of crossing steps with reduced electromigration force has been experimentally observed. A distinct difference has been observed in the way that $(1 \times 1)$ to $(7 \times 7)$ phase transition manifests itself on the $\mathrm{Si}(111)$ surfaces with a misorientation toward the $[11 \overline{2}]$ and $[\overline{1} \overline{1} 2]$ directions.
\end{abstract}

DOI: 10.1103/PhysRevB.82.153301

Dynamics of atomic steps on vicinal crystal surfaces and the phenomenon of step bunching have long been of great scientific interest. ${ }^{1}$ This is particularly true for the key surfaces such as $\mathrm{Si}(111)$ which is widely used in the semiconductor industry. This interest has however been particularly evident recently because the self-ordering and highly regular arrays of step bunches are strong candidates for the bottom-up fabricated templates sought for nanotechnological applications. ${ }^{2,3}$

Step bunching on $\mathrm{Si}(111)$ is induced by means of an electric heating current, passed through the sample along the surface. The process is driven by the surface drift of Si adatoms in the direction of the current flow. ${ }^{4}$ This drift results from the electromigration force acting on $\mathrm{Si}$ adatoms, which is defined as $F \equiv q_{\mathrm{eff}} E$, where $q_{\mathrm{eff}}$ is the $\mathrm{Si}$ adatom effective charge and $E$ is the applied electric field. While the influences of the annealing time, temperature, and current direction on the morphology of a bunched surface have been extensively investigated, ${ }^{5-12}$ the effects of the electromigration force on the step bunching process have never been explicitly isolated. There are currently four recognized temperature regimes for the step bunching on $\mathrm{Si}(111),{ }^{13}$ however in this study we operated in the regime II $\left(\sim 1050-1190{ }^{\circ} \mathrm{C}\right)$ where the step bunching takes place only when the heating current is driven in the step-up direction. In this regime, the step bunching process can be described within the framework of the transparent steps model, where the density of atomic step kinks is assumed to be low and most adatoms cross the steps without taking part in the exchange between the crystal phase and a layer of adatoms. ${ }^{8}$ The model predicts the expansion of step bunches with reduced electromigration force, however this has not been observed experimentally to date. The strength in our approach was that we could stabilize the temperature and current independently, i.e., for every value of current we could adjust the power of the external heater to bring the sample temperature to the desired set point value. This was done by means of a specially constructed heater offering an independent control of sample temperature by means of radiative heating as well as by direct current heating.

The experiments were performed at the base pressure of $2 \times 10^{-10}$ Torr. The step bunch morphology was produced by annealing series of miscut $\mathrm{Si}(111)$ samples at a fixed tem-
PACS number(s): 68.35.bg, 68.35.Rh, 68.35.Fx, 68.37.Ps

perature of $1130{ }^{\circ} \mathrm{C}$ while varying voltage across the sample. The maximum electric field of $3.9 \mathrm{~V} / \mathrm{cm}$ was applied when the set point temperature was achieved using exclusively direct current (dc) annealing. Correspondingly when the entire heating requirement was supplied by the radiative heater the applied electric field was zero.

Samples were prepared from $\mathrm{Si}(111) n$-type doped wafers (Siltronix, resistivity $\sim 1 \Omega \mathrm{cm}$ ) with a misorientation of $2.5^{\circ}$ toward the $[11 \overline{2}]$ direction or $2^{\circ}$ toward the $[\overline{1} \overline{1} 2]$ direction. The 0.525 -mm-thick wafers were cut into 20 $\times 1.5 \mathrm{~mm}^{2}$ rectangular strips with the long side along the miscut direction. The strips were mounted on a sample holder, between two electric contacts and lowered into an alumina crucible of an effusion cell (HTEZ by MBE Komponenten $\mathrm{GmbH}$ ) (Fig. 1). In this way, the voltage across the samples and the sample temperature could be controlled independently. To avoid sample contamination, we used an offfactory effusion cell that had not previously been used for any material evaporation. The sample temperature was extracted from the sample resistance, essentially using the sub-

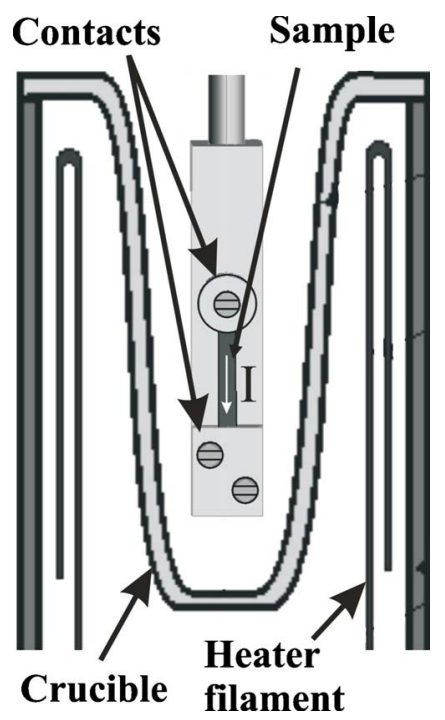

FIG. 1. (Color online) Schematic of a customized Mo sample holder placed into the heater. Applied electric field and sample temperature could be controlled independently in this way. 
strate as a resistance thermometer. The resistance vs temperature dependence was calibrated by heating the crucible to $1130{ }^{\circ} \mathrm{C}$ prior to the annealing procedure and measuring the resistance between the contacts by the two-point probe technique. The lead resistance was measured independently at the same temperature and taken into account when calculating electric field across the sample.

The annealing chamber was baked out at $110-130{ }^{\circ} \mathrm{C}$ for $12 \mathrm{~h}$. After the bakeout, the crucible was gradually heated to $650{ }^{\circ} \mathrm{C}$ and outgassed for $6 \mathrm{~h}$. Residual carbon was driven from the substrate surface into the bulk by repeated flash annealing to $1250{ }^{\circ} \mathrm{C}$ for $10 \mathrm{~s}$ using the dc current. The step bunches were formed by passing an in-plane dc current perpendicular to the atomic steps in the step-up direction for 12 $\mathrm{h}$ at $1130{ }^{\circ} \mathrm{C}$. Depending on the applied electric field, the crucible temperature was set so as to maintain the sample at $1130{ }^{\circ} \mathrm{C}$. Pressure during the annealing was below 5 $\times 10^{-9}$ Torr. The applied voltage was instantaneously switched off after annealing and the crucible was cooled down to $650{ }^{\circ} \mathrm{C}$ in about $2 \mathrm{~min}$. Such a short thermal annealing did not affect the shape of step bunches. ${ }^{6}$ The sample was maintained at $650{ }^{\circ} \mathrm{C}$ for $1 \mathrm{~h}$ before it was cooled down to room temperature and removed from the UHV for the ex situ atomic force microscopy (AFM) characterization

An experiment to determine the extent of carbon and oxygen contamination on the $\mathrm{Si}$ surface was conducted. A Si strip cut from the same wafer was placed into a Mo sample holder and annealed in an electron-beam heater in a separate chamber with a pressure of $\sim 6 \times 10^{-9}$ Torr. The steps of preannealing, flash annealing, and annealing were replicated and the surface cleanliness was analyzed by Auger electron spectroscopy (AES). The AES analysis showed that after flash annealing to $1250{ }^{\circ} \mathrm{C}$ the surface was free from carbon and oxygen contamination and remained clean after $12 \mathrm{~h}$ annealing at $1130{ }^{\circ} \mathrm{C}$.

The step bunching behavior at $1130{ }^{\circ} \mathrm{C}$ on the $\mathrm{Si}(111)$ surface with a misorientation of $2.5^{\circ}$ toward the [11ㄹ] direction is summarized in Fig. 2 which shows a series of AFM images of step bunch morphologies obtained at different voltages. The surface produced by a dc heating $(E$ $=3.9 \mathrm{~V} / \mathrm{cm}$ ) is characterized by $3.5-4.5 \mu \mathrm{m}$ wide terraces separated by $200-350 \mathrm{~nm}$ high step bunches aligned along the $[1 \overline{10}]$ direction [Fig. 2(a)]. The terraces are decorated by $\mathrm{S}$-shaped monatomic crossing steps aligned perpendicular to the step bunches. These crossing steps form a regular pattern on a number of terraces with a periodicity of 3-6 $\mu \mathrm{m}$ along the $[1 \overline{10}]$ direction with a greater periodicity for the steps forming on wider terraces. For a fixed annealing time, the crossing steps gradually elongate with a decreasing applied electric field, as can be seen in Fig. 2(b). The steps are curved in a long $\mathrm{S}$ shape and run over $2-3 \mu \mathrm{m}$ along the [1] 0$]$ direction with a periodicity of $0.5-1.5 \mu \mathrm{m}$.

A notable transformation in surface morphology is evident in Fig. 2(c), which shows the step bunched surface obtained at $E=1 \mathrm{~V} / \mathrm{cm}$. The periodicity of crossing steps along the $[1 \overline{1} 0]$ direction is reduced to $500-950 \mathrm{~nm}$. These crossing steps run over a distance of 8-10 $\mu \mathrm{m}$ and are decorated by chains of triangular islands. Figure 2(d) shows the result of annealing at $E=0.9 \mathrm{~V} / \mathrm{cm}$. The step bunches expand fur-

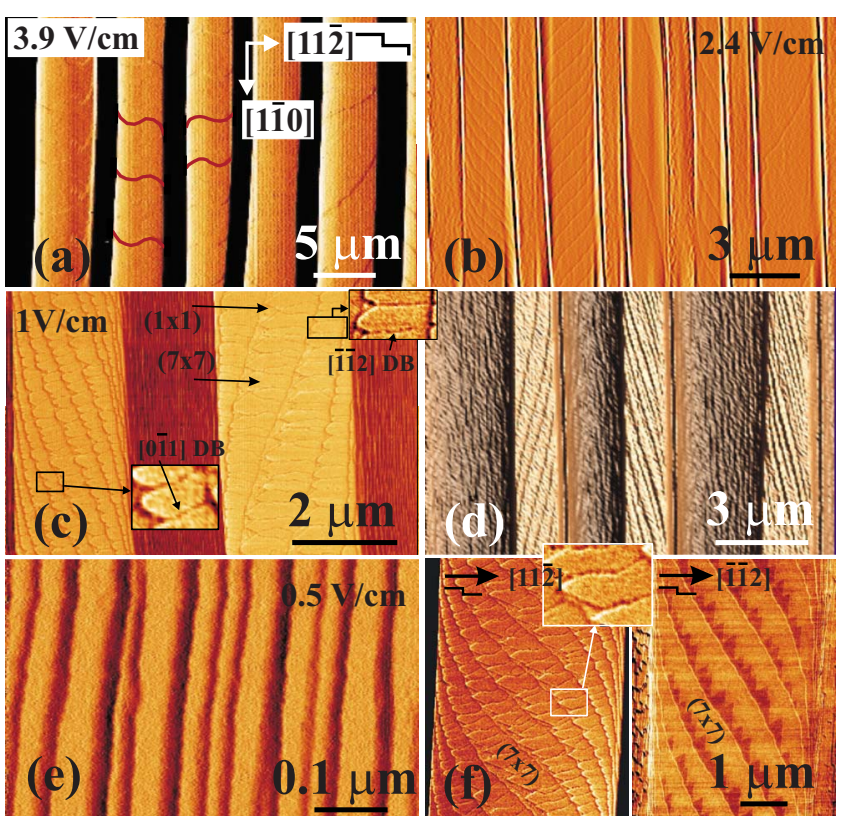

FIG. 2. (Color online) The step bunching behavior on a $\mathrm{Si}(111)$ surface at $1130{ }^{\circ} \mathrm{C}$ at different electromigration forces. The surface is off cut $2.5^{\circ}$ toward the $[11 \overline{2}]$ direction. The direction of a miscut is from left to right in all images. The heating current was maintained in the step-up direction throughout the whole annealing process. (a) Differentiated AFM image of a step bunched $\mathrm{Si}(111)$ surface obtained by dc annealing; $E=3.9 \mathrm{~V} / \mathrm{cm}$, annealing current $I$ $=2.4 \mathrm{~A}$; the shape of selected crossing steps have been highlighted for clarity. (b) Differentiated AFM image of Si(111) after annealing at $E=2.4 \mathrm{~V} / \mathrm{cm}$ and $I=1.5 \mathrm{~A}$. (c) Phase AFM image of $\mathrm{Si}(111)$ after annealing at $E=1 \mathrm{~V} / \mathrm{cm}$ and $I=0.6 \mathrm{~A}$. Crossing steps are decorated by chains of unreconstructed $(1 \times 1)$ triangular islands while the rest of terrace is a $(7 \times 7)$ reconstructed surface; the $(7$ $\times 7)$ surface consists of $(7 \times 7)$ domains separated by DBs; the insets show the domains boundaries oriented in the [011] and [ $\overline{1} \overline{1} 2]$ directions. (d) Derivative AFM image demonstrating that the step bunches expand and occupy most of the bunched surface after annealing at $E=0.9 \mathrm{~V} / \mathrm{cm}$ and $I=0.55$ A. (e) Derivative AFM image of $\mathrm{Si}(111)$ after annealing at $E=0.5 \mathrm{~V} / \mathrm{cm}$ and $I=0.3 \mathrm{~A}$; step bunching process ceases at the applied electric fields lower than 0.5 $\mathrm{V} / \mathrm{cm}$. (f) On the left is an enlarged terrace of the sample shown in Fig. 2(c); the inset shows a domain boundary that consists of the [0 $\overline{1} 1]$ and [ $\overline{1} \overline{1} 2]$ segments; on the right is a terrace of a $\mathrm{Si}(111)$

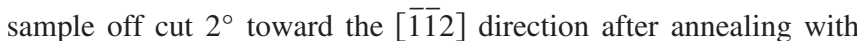
$E=1.5 \mathrm{~V} / \mathrm{cm}$ and $I=1.1 \mathrm{~A}$; the $(7 \times 7)$ areas are continuous and uninterrupted by domain boundaries.

ther due to the decrease in the applied field ${ }^{8}$ and occupy $\sim 70 \%$ of the bunched surface while the terrace width is decreased to $1.6-1.8 \mu \mathrm{m}$. The crossing steps run over a distance of 4-5 $\mu \mathrm{m}$, and the distance between them is reduced to $40-120 \mathrm{~nm}$. This is the systematic observation of the theoretically predicted increase in the number of crossing steps with reduced electromigration force. ${ }^{14,15}$ Sato and Uwaha demonstrated that crossing steps form due to the sublimation of adatoms and terraces are free of crossing steps when evaporation is negligible. ${ }^{14}$ In our experiment when a weaker electromigration force is applied to Si adatoms, they drift at lower velocity and evaporate before they cross the terrace 
and take part in the exchange between the crystal phase and the layer of migrating adatoms. The increased number of crossing step under the weak drift conditions was also theoretically predicted by Popkov and Krug at around 900 and $1250{ }^{\circ} \mathrm{C} .{ }^{30}$

At the critical electric field $E_{\mathrm{cr}}=0.5 \mathrm{~V} / \mathrm{cm}$, the electromigration force is no longer sufficient to initiate the step bunching process with coarsening, where the number of steps in the bunch gradually increases with the sublimation time. As a result, the large step bunches comprising hundreds of atomic steps no longer appear to be a favorable surface configuration and the formation of the traveling step density wave instability is instead observed on the surface [Fig. 2(e)]. This instability differs from the electromigrationinduced step bunching in the sense that the step density waves contain a relatively small number of steps (compared to the number of steps in coarsening step bunches) and this number is not affected by the duration of sublimation. However, even below $E_{\mathrm{cr}}$ the applied electric field is still found to have an impact on the step dynamics. For example, at $E_{\mathrm{cr}}$ $=0.5 \mathrm{~V} / \mathrm{cm}$, the surface is characterized by $1.5-3.5 \mathrm{~nm}$ high step bunches (5-12 atomic steps) separating 25-65-nm-wide terraces while reduction in electric field below $0.3 \mathrm{~V} / \mathrm{cm}$ produced a surface covered by arrays of double and triple atomic steps. This observation is in agreement with the results of recent numerical modeling of the step bunching process which theoretically predicted the existence of a critical electromigration force as well as the formation of step density waves with relatively weak or zero electromigration. ${ }^{16,17}$ These new models extend beyond the quasistatic approximation of earlier models ${ }^{8,9}$ and take into account the movement of atomic steps as well as a different rate of adatom surface diffusion, step crossing, and attachment-detachment at the steps. ${ }^{16,17}$ However, the formation of step density waves in these simulations ${ }^{16}$ is observed at an electromigration force which is two orders of magnitude larger than the $F_{\mathrm{cr}}=2.7$ $\times 10^{-18} \mathrm{~N}$ deduced in our experiment on the assumption that effective charge $q_{\text {eff }}$ of a $\mathrm{Si}$ adatom is equal to $1 / 3$ of the elementary electric charge. ${ }^{10}$

Crystallographic off-cut direction was found to affect final surface morphology on the terraces. The triangular islands along the crossing step edges apparent in Fig. 2(c) are similar to structures previously observed upon quenching $\mathrm{Si}(111)$ from $1250{ }^{\circ} \mathrm{C}$ to about $860{ }^{\circ} \mathrm{C}$ (Refs. 18-21) where the surface undergoes a $(1 \times 1)$ to $(7 \times 7)$ phase transition. ${ }^{22-27}$ The islands were identified as $(1 \times 1)$ domains and the remainder as a $(7 \times 7)$ reconstructed surface. Upon oxidation in ambient conditions, the $(1 \times 1)$ islands appear $\sim 0.2-0.3 \mathrm{~nm}$ higher than the $(7 \times 7)$ reconstructed parts of terraces in the AFM images. ${ }^{28}$ Figure $2(\mathrm{f})$ shows that there is a distinct difference in how the $(1 \times 1)$ to $(7 \times 7)$ phase transition manifests itself on the [ $\overline{1} \overline{1} 2]$ and $[11 \overline{2}]$ miscut surfaces. On surfaces off cut toward the $[11 \overline{2}]$ direction the $(7 \times 7)$ triangular domains nucleate at the upper step edges and propagate into $(1 \times 1)$ terraces [Figs. 2(c) and 2(f) left]. ${ }^{18,25,28,29}$ This $(7 \times 7)$ growth ceases upon reaching the next crossing step leaving the triangular unreconstructed islands of $(1 \times 1)$ phase along the lower step edges. ${ }^{21,30}$ The origin of the corner led expansion

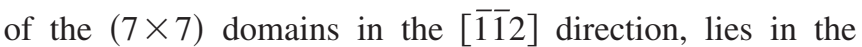

threefold symmetry of the $\mathrm{Si}(111)$ surface and the two unequivalent triangular subunits of the $(7 \times 7)$ unit cell. The $(7 \times 7)$ unit cell is composed of faulted and unfaulted triangular halves, with the faulted half always pointed in one of three $\langle\overline{1} \overline{1} 2\rangle$ directions. It is more energetically favorable for $(7 \times 7)$ domains to be bordered by faulted segments, thus as they grow their orientation is preserved. ${ }^{30}$ Expansion parallel to the direction of crossing steps results in the formation of parallel domain boundaries (DBs) between neighboring (7 $\times 7)$ domains and distorts their original triangular profile. ${ }^{30-32}$ Some of the domain boundaries align in either [0 $\overline{1} 1]$ or $[\overline{1} \overline{1} 2]$ direction individually [Fig. 2(c)] while others consist of combinations of segments of both directions [Fig. 2(f) left]. Only [1-12] domain boundaries were present in proximity to the step bunches where the crossing steps align close to the $[1 \overline{1} 0]$ direction.

Correspondingly, on surfaces off cut toward the [1-̄2] direction, the straight edges of the $(7 \times 7)$ phase triangles expand from the upper step edge as a continuous band in the

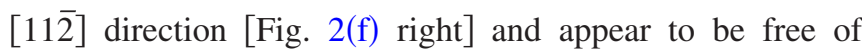
domain boundaries. This growth halts when a corner of a $(7 \times 7)$ triangle reaches the next crossing step. Thus a saw tooth chain of $(1 \times 1)$ islands is created due to the mismatched orientation of the crossing steps with respect to the growth direction of the $(7 \times 7)$ phase. Accordingly, as the crossing steps tend toward the step bunches they align with the edge of the $(7 \times 7)$ expanding phase and the size of the $(1 \times 1)$ islands diminishes until they disappear and the terrace is covered exclusively by the $(7 \times 7)$ reconstruction in the vicinity of step bunches.

In conclusion, we studied the effects of the electromigration force on the step bunching process on a miscut $\mathrm{Si}(111)$ surface in the temperature regime II $\left(\sim 1050-1190{ }^{\circ} \mathrm{C}\right)$. We observed experimentally that the reduction in applied inplane electric field results in the expansion of step bunches to such extent that they eventually occupy most of the bunched surface. Applying an in-plane electric field of $E=0.5 \mathrm{~V} / \mathrm{cm}$ or weaker results in cessation of the step bunching process and creates traveling step density waves. Crossing steps assume a long $\mathrm{S}$ shape and run along the terraces over a distance of several micrometers. As the applied electric field decreases, the distance between the crossing steps is reduced to $\sim 100 \mathrm{~nm}$ or less as opposed to $3-6 \mu \mathrm{m}$ in the case of the annealing by entirely direct current. We observed a distinct difference in how the $(1 \times 1)$ to $(7 \times 7)$ phase transition manifests itself on the terraces bordered by the crossing

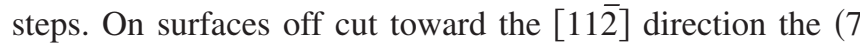
$\times 7)$ phase is seen as $(7 \times 7)$ domains separated by domain boundaries, contrary to the surfaces off cut toward the [112] direction where the $(7 \times 7)$ phase appears as a continuous band uninterrupted by the phase boundaries.

The authors would like to thank S. Stoyanov for his helpful discussion. The financial support of Science Foundation Ireland, Contract No. 06-IN.1/I91 is gratefully acknowledged. 
*Corresponding author; usovv@tcd.ie

${ }^{1} \mathrm{~J}$. Krug, in Multiscale Modeling in Epitaxial Growth, edited by A. Voigt, Vol. 149 of International Series of Numerical Mathematics (Birkhauser, Verlag, 2005), p. 69.

${ }^{2}$ I. V. Shvets, H. C. Wu, V. Usov, F. Cuccureddu, S. K. Arora, and S. Murphy, Appl. Phys. Lett. 92, 023107 (2008).

${ }^{3}$ F. Cuccureddu, V. Usov, S. Murphy, C. O'Coileain, and I. V. Shvets, Rev. Sci. Instrum. 79, 053907 (2008).

${ }^{4}$ M. Degawa, H. Minoda, Y. Tanishiro, and K. Yagi, Surf. Sci. 461, L528 (2000).

${ }^{5}$ A. Latyshev, A. Aseev, A. Krasilnikov, and S. Stenin, Surf. Sci. 213, 157 (1989).

${ }^{6}$ E. S. Fu, M. D. Johnson, D. J. Liu, J. D. Weeks, and E. D. Williams, Phys. Rev. Lett. 77, 1091 (1996).

${ }^{7}$ J. Krug, V. Tonchev, S. Stoyanov, and A. Pimpinelli, Phys. Rev. B 71, 045412 (2005).

${ }^{8}$ S. Stoyanov, Surf. Sci. 416, 200 (1998).

${ }^{9}$ S. Stoyanov and V. Tonchev, Phys. Rev. B 58, 1590 (1998).

${ }^{10}$ K. Fujita, M. Ichikawa, and S. S. Stoyanov, Phys. Rev. B 60, 16006 (1999).

${ }^{11}$ Y. Homma and N. Aizawa, Phys. Rev. B 62, 8323 (2000).

${ }^{12}$ B. J. Gibbons, S. Schaepe, and J. P. Pelz, Surf. Sci. 600, 2417 (2006).

${ }^{13}$ F. Leroy, D. Karashanova, M. Dufay, J. M. Debierre, T. Frisch, J. J. Métois, and P. Müller, Surf. Sci. 603, 507 (2009).

${ }^{14}$ M. Sato and M. Uwaha, Surf. Sci. 442, 318 (1999).

${ }^{15}$ V. Popkov and J. Krug, Phys. Rev. B 73, 235430 (2006).

${ }^{16}$ B. Ranguelov and S. Stoyanov, Phys. Rev. B 77, 205406 (2008).

${ }^{17}$ B. Ranguelov and S. Stoyanov, Surf. Sci. 603, 2907 (2009).
${ }^{18}$ N. Osakabe, Y. Tanishiro, K. Yagi, and G. Honjo, Surf. Sci. 109, 353 (1981).

${ }^{19}$ N. Aizawa and Y. Homma, Surf. Sci. 340, 101 (1995).

${ }^{20}$ M. Hoshino, Y. Shigeta, K. Ogawa, and Y. Homma, Surf. Sci. 365, 29 (1996).

${ }^{21}$ H. Hibino, Y. Homma, and T. Ogino, Phys. Rev. B 58, R7500 (1998).

${ }^{22}$ J. J. Lander, Surf. Sci. 1, 125 (1964).

${ }^{23}$ R. J. Phaneuf, E. D. Williams, and N. C. Bartelt, Phys. Rev. B 38, 1984 (1988).

${ }^{24}$ S. Hasegawa, Y. Nagai, T. Oonishi, and S. Ino, Phys. Rev. B 47, 9903 (1993).

${ }^{25}$ A. V. Latyshev, A. B. Krasilnikov, A. L. Aseev, L. V. Sokolov, and S. I. Stenin, Surf. Sci. 254, 90 (1991).

${ }^{26}$ Y. N. Yang and E. D. Williams, Phys. Rev. Lett. 72, 1862 (1994).

${ }^{27}$ U. Höfer, L. Li, G. A. Ratzlaff, and T. F. Heinz, Phys. Rev. B 52, 5264 (1995).

${ }^{28}$ K. Nakayama and T. Shiota, Surf. Interface Anal. 40, 885 (2008).

${ }^{29}$ S. Kitamura, T. Sato, and M. Iwatsuki, Nature (London) 351, 215 (1991).

${ }^{30}$ C. W. Hu, H. Hibino, T. Ogino, and I. S. T. Tsong, Surf. Sci. 487, 191 (2001).

${ }^{31}$ M. Itoh, H. Tanaka, Y. Watanabe, M. Udagawa, and I. Sumita, Phys. Rev. B 47, 2216 (1993).

${ }^{32}$ Y. F. Zhao, H. Q. Yang, J. N. Gao, Z. Q. Xue, and S. J. Pang, Phys. Rev. B 58, 13824 (1998). 